\title{
OBSERVATIONS OF THE BOWEN FLUORESCENCE MECHANISM AND CHARGE TRANSFER IN PLANETARY \\ NEBULAE I.
}

\author{
XIAO-WEI LIU and JOHN DANZIGER \\ European Southern Observatory, Garching bei München, FRG
}

Deep, medium resolution, long-slit spectrophotometric data have been taken for a number of high-excitation planetary nebulae, covering a wavelength range from $3100 \AA$ to $7200 \AA$ with some selected regions observed at higher resolution. For about half the objects, the whole optical region has been observed, from $3100 \AA$ to $11600 \AA$. Accurate flux calibration is achieved over this whole wavelength range. These data allow a detailed quantitative study of the Bowen fluorescence mechanis$m$ and the charge transfer reaction $\mathrm{O}^{3+}\left({ }^{2} \mathrm{P}_{3 / 2,1 / 2}\right)+\mathrm{H}^{0}-\mathrm{O}^{2+}\left({ }^{2 s_{\mathbf{a}}^{\prime}+1} \mathrm{~L}_{\mathrm{a} \mathrm{j}_{\mathbf{a}}^{\prime}}^{\prime}\right)+\mathrm{H}^{+}$in planetary nebulae, the primary goal of this program. In this paper measurements of $\mathrm{O}^{2+}$ Bowen fluorescence and charge transfer lines are presented. We show that $L S$ coupling fails for the $\mathrm{O}^{2+}$ permitted transitions studied in this program and intermediate coupling may be a better assumption. Efficiencies of the Bowen fluorescence mechanism are derived for 15 objects, and a wide range of possible values is apparent. There is a remarkable linear positive correlation between the Bowen efficiency and the fractional abundance of oxygen in the ionization stage of $\mathrm{O}^{2+}$ and the fractional abundance of helium in the form of $\mathrm{He}^{+}$. Evidence that the Bowen efficiency is anticorrelated with the electron temperature, as first noted by Likkel and Aller, is established. The Bowen efficiency drops substantially when the nebular expansion velocity $2 V_{e x p}\left(\mathrm{O}^{2+}\right)>55 \mathrm{~km} / \mathrm{sec}$. For lower expansion velocities there is no detectable correlation between these two quantities. There are no observable differences in Bowen efficiency among objects of different morphological type nor between objects excited by stars of different spectral types as suggested by Likkel and Aller.

The OIII $\lambda 3261, \lambda 3265, \lambda 3267$ lines are found to originate from radiative and dielectronic recombination. $\mathrm{O}^{3+}$ abundances are derived for some objects using these lines. 